\title{
DEVELOPMENT OF ANTIFIBRINOLYTIC PROPERTIES IN BLOOD OF PATIENTS WITH RHEUMATIC FEVER, CHRONIC INFEC- TIVE ARTHRITIS AND BACTERIAL ENDOCARDITIS ${ }^{1}$
}

\author{
BY ERIK WAALER
}

(From the University Medical Clinic B. of Rikshospitalet, Oslo, Norway)

\author{
(Received for publication September 8, 1936)
}

The ability of the hemolytic streptococci of the beta type to dissolve human fibrin clot has been demonstrated by Tillett and Garner (1). They have also found (2) that the blood of patients convalescent from acute hemolytic streptococcal infections was highly resistant to the fibrinolytic principle. This antifibrinolytic property of the blood has been tested by several authors in various infections due to hemolytic streptococci. Tillett (3) found that the antifibrinolytic factor was demonstrable in the blood of seventy-five per cent of patients who had recovered from acute streptococcal infections. In rheumatic fever the antifibrinolytic property seemed to follow the acute upper respiratory infection. Hadfield, Magee, and Perry (4) reported that the plasma clot was resistant during the active stage of the disease in rheumatic fever. The antifibrinolysin reaction has also been used by Myers, Keefer and Holmes (5), who found positive reactions in rheumatic fever, but negative reactions in rheumatoid arthritis.

In a previous paper (6) the author reported the results of antifibrinolysin tests in which Tillet and Garner's technic (1) was used. The results were graded according to the rapidity with which dissolution took place. Each plasma was tested against 5 strains of streptococci with varying fibrinolytic ability; only one dilution of active culture was used. With this technic we were able to demonstrate positive antifibrinolysin reactions in 5 cases of acute polyarthritis, while 5 others were negative in the active stage of the disease. In a few cases of rheumatoid arthritis we also found resistance to the fibrinolytic principle. We therefore decided to try another. technic in order, if possible, to demonstrate weaker positive reactions.

\section{MATERIALS AND METHODS}

We have studied 7 cases of acute polyarthritis, 24 cases of chronic infective polyarthritis, and 5 cases of bacterial

1 This study was made possible by the aid of the Else and Marie Mustad Fund. endocarditis. Most of the patients have been under treatment at the Medical Department B, Rikshospitalet. Some tests were carried out with samples of blood sent from other departments and hospitals. In order to state the "normal" reaction we have performed tests on 39 healthy adults, chosen among students, technicians and members of the hospital staff. In this group we selected those who were healthy at the time the test was made, and who gave no history of acute infection during the past three months.

One strain of hemolytic streptococcus of the beta type has been employed in all the tests. The strain was derived from the throat of a patient suffering from rheumatic fever. It was highly active, and its fibrinolytic activity did not seem to change during the 6 months required for the tests. Fresh 18-hour old cultures were always used.

Numerous trials with varying amounts of the different agents have been made. At first we tested the ability of a serum, from individuals whose plasma was resistant, to render normal plasma clot insusceptible. Small amounts of fibrinolytic culture were used, and the method was in many respect similar to the antistreptolysin titration of Todd (7). This method proved unsuitable, however, as the serum from resistant individuals conferred only a small amount of antifibrinolytic property upon normal plasma clots. We have found it more convenient to put up a series of dilutions of the active culture and to test their lytic effect upon a constant amount of plasma. In this way we were able to determine the smallest dose of active culture capable of causing lysis of the plasma clot. Thus, a more accurate grading of the resistance is made possible, and it is an easy matter to follow variations in this resistance from time to time.

We found that the peptone used in the broth, in some way affects the clot formation. Parke Davis peptone in particular made the coagulation of plasma incomplete, while Cogit peptone had a less disturbing effect. Furthermore, when Parke Davis peptone was used, the coagulated plasma was rolled up into a compact mass, leaving the rest of the tube's contents completely fluid. Owing to this retraction of the coagulum, it was often difficult to see the difference between the positive and negative reactions. After some trial experiments, we found that the tests were read more easily when Ringer's solution was used instead of normal saline. Solid coagulation appeared rapidly, and even after 20 hours' incubation, the tubes could be inverted without affecting the solid clot which adhered to the bottom and the sides of the tube. In the tubes where dissolution occurred, all evidence of clotted plasma disappeared, and the contents 
became completely fluid. In a few tubes containing a small amount of active culture the liquefaction might be somewhat incomplete, but in any case there was a great difference between these tubes and the tubes in which the contents were solid and resistant. Thus, there was a distinct difference between the positive and the negative reactions. With this procedure we were able to use Parke Davis peptone throughout the work.

The technical procedures have in all other respects been those recommended by Tillett and Garner (1). We have used oxalated plasma, clotted with calcium chloride. The amount of anticoagulant, potassium oxalate, was 0.02 gram to $10 \mathrm{cc}$. blood. In the tests, $0.2 \mathrm{cc}$. plasma was used, and the reactions were always carried out within 3 hours after withdrawal of blood. Of a 0.25 per cent solution of $\mathrm{CaCl}_{2}$ in 0.85 per cent salt solution $0.25 \mathrm{cc}$. was used as coagulant.

Description of test. Amounts of an 18-hour culture ranging from $0.1 \mathrm{cc}$. to $0.0000125 \mathrm{cc}$. respectively, were mixed with $0.5 \mathrm{cc}$. of Ringer's solution (see Figure 1). To each tube was added $0.8 \mathrm{cc}$. Ringer's solution, and then $0.2 \mathrm{cc}$. plasma. The coagulant, $0.25 \mathrm{cc}$. of the $\mathrm{CaCl}_{2}$ solution, was then added and well mixed. The tubes were incubated at $37^{\circ} \mathrm{C}$.

A clot formed within a few minutes, and the liquefaction of a susceptible plasma clot started after $1 / 2$ to 1 hour in the tubes containing the highest concentrations of active culture. All tests were read after 4 and after 20 hours of incubation. The results after 20 hours of incubation proved to be the most reliable, and in this paper only the 20-hour results are given.

\section{Normal controls}

Thirty-nine " normal" subjects were tested. The results are shown in Figure 1, those from individuals yielding the same type of reaction being assembled in one column. In 16 of the normal subjects only the control tubes were left unaffected by the lytic principle. In two of these, the contents of all tubes containing active culture were completely liquefied. In the remaining 14 , complete dissolution did not occur in a certain number of tubes (varying from 2 to 5 ), which contained the smallest amount of culture. Eleven subjects showed no dissolution in the tube containing the smallest amount of active culture $(0.0000125$ cc. $)$. In two of these subjects the plasma was completely liquefied in all other tubes, while the remaining 9 subjects gave incomplete liquefaction in a varying number of the tubes (from 2 to 4 ) containing the smaller amounts of active culture. Seven individuals were resistant to the doses $0.0000125 \mathrm{cc}$. and $0.000025 \mathrm{cc}$. The

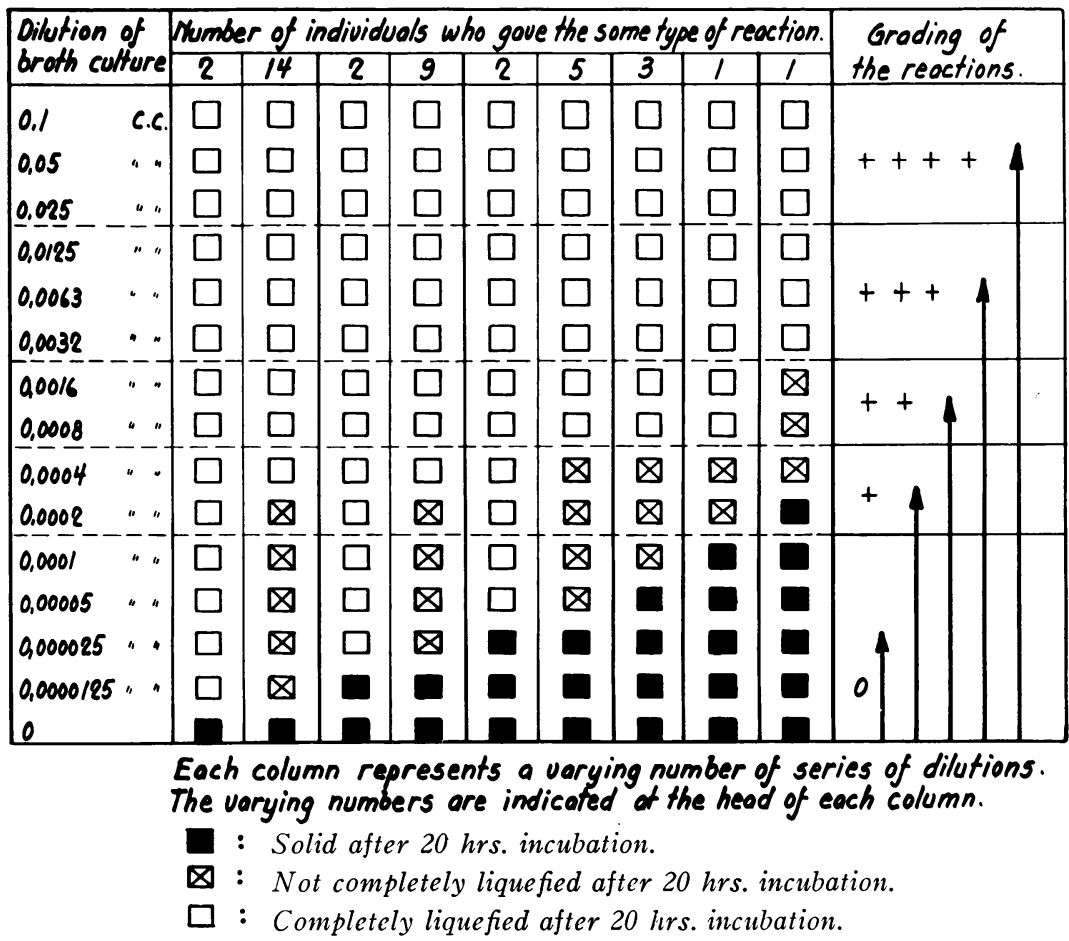

Fig. 1. Resistance to Fibrinolytic Activity in Normal Individuals. 
plasma clots from the majority of the cases (34) were thus very susceptible; those from 5 were somewhat more resistant. Four of the latter patients could not be said to have had any streptococcal infections. The clot from the fifth subject, whose reaction is given last in the chart, was completely resistant up to the dose $0.0002 \mathrm{cc}$. He was more thoroughly examined, and admitted that his tonsils had been removed one and a half months previously, on account of repeated attacks of sore throat. This was probably the reason for the high resistance of his clot.

The reactions are graded as follows (Figure $1)$. In the patients whose plasma clot was resistant to all dilutions of active streptococcal culture broth up to the dose $0.025 \mathrm{cc}$. or higher, the designation is ++++ . Resistance only up to 0.0125 cc., 0.0063 cc., or 0.0032 cc. is termed +++ ; up to $0.0016 \mathrm{cc}$. or $0.0008 \mathrm{cc}$. ++ ; and resistance only up to $0.0004 \mathrm{cc}$. or $0.0002 \mathrm{cc}$. is designated + . The reaction is regarded as negative or normal when the clot remains intact only in the presence of $0.0001 \mathrm{cc}$. or less of the broth culture of streptococcus used. The end point was read from the tubes in which the contents were completely solid after 20 hours of incubation, and not from those in which incomplete liquefaction took place. There was thus only one of the 39 normal subjects who gave a weak positive antifibrinolysin reaction. The value of this rough grading is naturally restricted; for this reason we have, to a great extent, reproduced charts demonstrating the reactions obtained with plasma from the various patients.

\section{Acute polyarthritis}

Plasma clots from 7 patients with acute polyarthritis were examined. All of the patients had had sore throat or more definite angina 1 to 2 weeks before the joint symptoms appeared. The antifibrinolysin reactions of the plasma from these patients are given in Figures 2, 3 and 4.

Patient Number 1, E. J. (Figure 2), 31-year old woman, went through a severe attack. Several joints were affected, but no carditis developed. On the 13 th and 29 th day after the onset of the first joint symptoms, her plasma clot was completely resistant. Following improvement, her plasma became more susceptible. She was discharged without any symptoms, and with a completely normal fibrinolysin reaction.

Patient Number 2, P. R. (Figure 2), 18-year old boy, had a severe attack. Several joints were swollen and tender. He had a mild carditis, with prolonged conduction time and deviation of the ST line which disappeared during the course of the disease. Thirty and 44 days after the onset of the joint symptoms the anti-

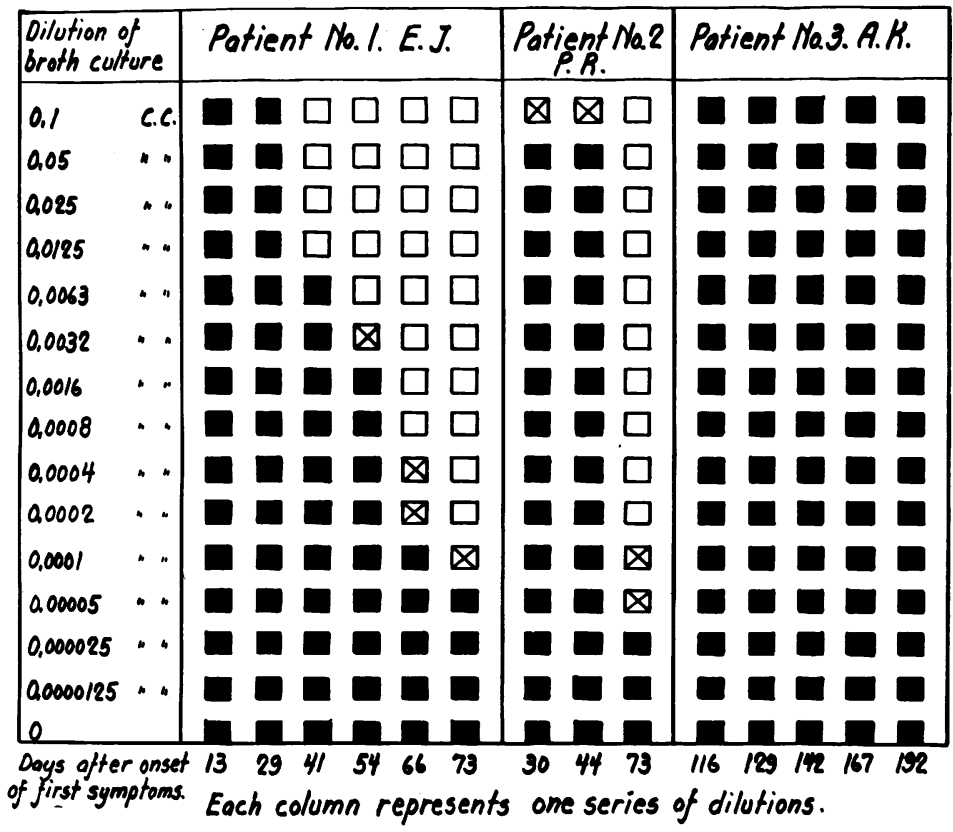

Fig. 2. Resistance to Fibrinolytic Activity in Rheumatic Fever. 


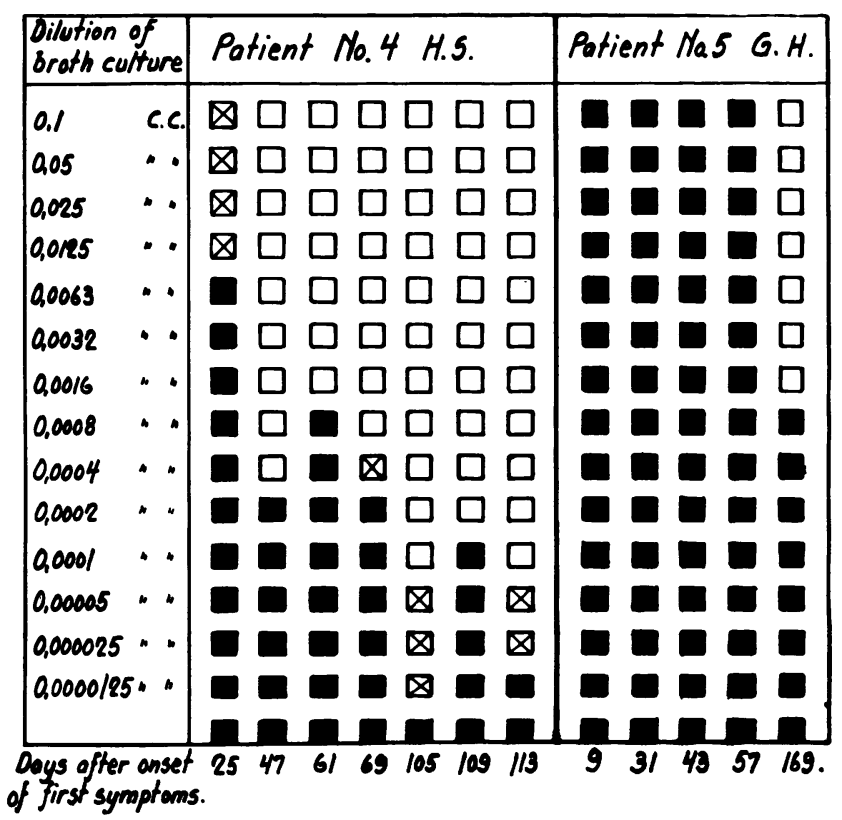

Fig. 3. Resistance to Fibrinolytic Activity in Rheumatic Fever.

fibrinolysin reaction was completely positive. After 73 days his plasma clot was susceptible, and he was discharged without any symptoms.

Patient Number 3, A. K. (Figure 3), 12-year old girl, had a very severe attack with migrating polyarthritis, and severe continuous carditis. She died after half a year's illness. The plasma clot from this patient was completely resistant during the time she was under the author's observation. The last test was performed 14 days before death.

Patient Number 4, H. S. (Figure 3), 36-year old man, had a history of rheumatic disease at the age of 9 , with three recrudescences. The present was his fifth attack, and of average severity with a mild carditis; he had prolonged conduction time which became normal during the course of the disease. On the 25th day of disease his plasma clot was resistant though not completely so. The reaction was weaker on the 47 th day. The plasma was, however, more resistant after 61 days. This was probably due to a mild recrudescence following the extraction of several infected teeth. After this, the reaction became weaker, and on the 105th day the clot was normally susceptible. Tonsillectomy was carried out and three days afterwards (on the 109th day) there was a slight rise in resistance of the plasma clot to fibrinolysis. Correspondingly he had a mild recrudescence of his disease of short duration.

Patient Number 5, G. H. (Figure 3), 25-year old man, experienced a typical attack of rheumatic fever at the age of 12 and developed heart failure. He was admitted with a history of insidious onset of the joint symptoms, and signs of severe continuous myocarditis and endocarditis. He stayed in the hospital from the 9 th to the 57 th day of disease, and during this time the antifibrinolysin reaction was completely positive. After 5 months (169th day) his plasma clot was less resistant. During the time he had stayed at home his improvement had been steady and marked.

Patient Number 6, A. T. (Figure 4), 34-year old woman, was admitted with a severe acute polyarthritis, the course of which proved to be insidious. No carditis was found. On the 4th and 8th day of the disease her plasma clot was somewhat more resistant than in normal subjects. However, the reaction was distinctly positive. The infection of the throat appeared 12 days before the first test was performed.

Patient Number 7, A. R. (Figure 4), 25-year old man, had a very mild and vague rheumatic attack of short duration without carditis. During the course of disease the antifibrinolysin reaction was completely normal. In this patient the first reaction was carried out 20 days after the manifestation of the angina.

In patients Numbers $1,2,4$, and 5 the reactions were completely or almost completely positive about 4 weeks after. the onset of the first joint symptoms. At this time the plasma clot of patient Number 6 had acquired normal susceptibility, whereas the reaction at an earlier stage of the disease was weakly positive. After 4 weeks, the polyarthritis in patient Number 6 was distinctly active. Her condition was, however, better, and 


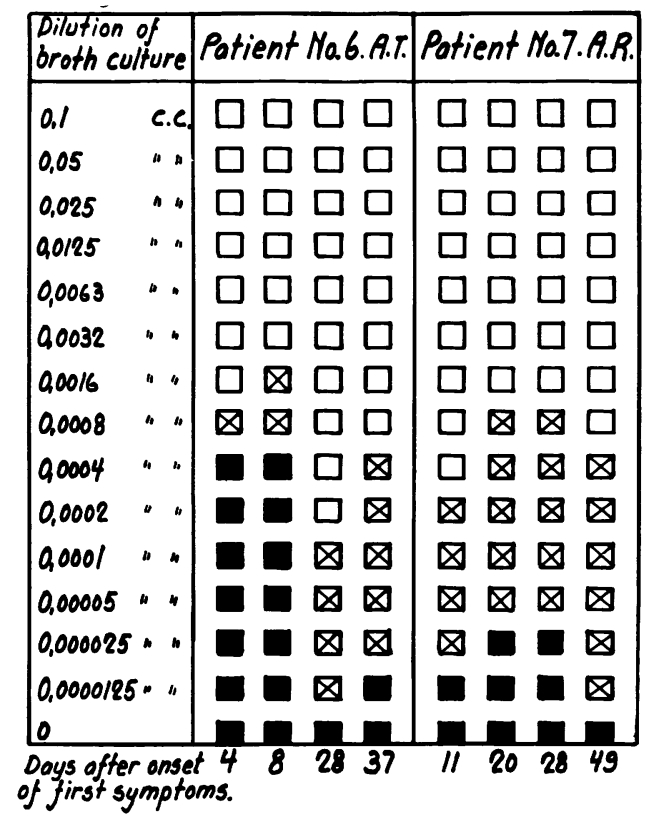

Fig. 4. Resistance to Fibrinolytic Activity in Rheumatic Fever.

correspondingly the weakly positive reaction disappeared.

In summary, it appears that extremely high resistance of plasma clots is associated with intense rheumatic activity. Thus, the clots from the two patients, Numbers 3 and 5, with the most severe disease, were most resistant to fibrinolysis. When the rheumatic manifestations disappeared, the antifibrinolysin reaction of the plasma returned to a normal level.

Throat cultures were studied from patients Numbers 1, 3, 4, 5, 6, and 7. In patient Number 1 , no hemolytic strain was found. In patients Numbers 3, 4, and 5, on the other hand, hemolytic strains of streptococci were recovered from the throats, and these strains were actively fibrinolytic. In the last two patients, Numbers 6 and 7 , no fibrinolytic strains were found, in spite of the fact that the tonsils were swabbed and cultured on blood agar plates six times and that a culture was made from the interior of the tonsils after their removal. From patient Number 6 we recovered no hemolytic strains, and the hemolytic strains recovered from patient Number 7 possessed no fibrinolytic activity. It is possible that these observations may be helpful in explaining the variations in reactions. It may thus be as- sumed that the patients who harbor in their throats the strains with the most extreme fibrinolytic activity will give the most definite immune response and the most active disease picture. Our data are not sufficiently extensive, however, to justify an opinion on this point.

\section{Chronic infective arthritis}

Twenty-four cases were studied. Five of the patients were children, probably belonging to the group of Still's disease. The other 19 were adults, and according to the clinical picture, they must be characterized as rheumatoid (atrophic) arthritis. Several of the patients had suffered from their disease for one year or longer, and all of them had active symptoms at the time the tests were performed. Roentgen examinations of the affected joints showed calcareous atrophy to a varying degree. In the more advanced cases, slight deformation of the joint surfaces had developed with formation of osteophytes. Seven of the patients were examined several times during the course of the disease (Figures 5 and 6).

Patient Number 8, H. A. (Figure 5), 31-year old man, for one year had had rheumatoid arthritis. Several joints were affected; no carditis was noted. $\mathrm{He}$ was febrile with a sedimentation rate of the red blood corpuscles of about $120 \mathrm{~mm}$. after 1 hour. On March 1st and April 4th the antifibrinolysin reaction was positive, whereas the clot was normally susceptible on May 28th. During this time he had been treated with streptococcal vaccine, with no change in symptoms.

Patient Number 9, G. N. (Figure 5), 45-year old woman, was admitted with a story of polyarthritis of varying intensity for 6 months. Mainly the hips were affected. On arrival she was febrile, sedimentation rate $35 \mathrm{~mm}$., no carditis. The first test on the plasma of this patient was positive. At the time of the last test the disease was less active, and her clot had normal susceptibility.

Patient Number 10, E. R. (Figure 5), 51-year old man, during 15 years was known to have had several attacks of fever and migrating polyarthritis. In the hospital he was subfebrile with a sedimentation rate of about $50 \mathrm{~mm}$. $\mathrm{He}$ had severe carditis (heart block). The two tests carried out with the blood from this patient gave positive reactions. The disease was apparently less active at the time of the last test, and, correspondingly, the plasma clot was more susceptible.

Patient Number 11, K. M. (Figure 5), 33-year old woman, for two years had had joint symptoms of varying intensity. She was subfebrile, had no carditis, and the sedimentation rate on admission was $110 \mathrm{~mm}$. It became normal during the time she stayed in the hos- 


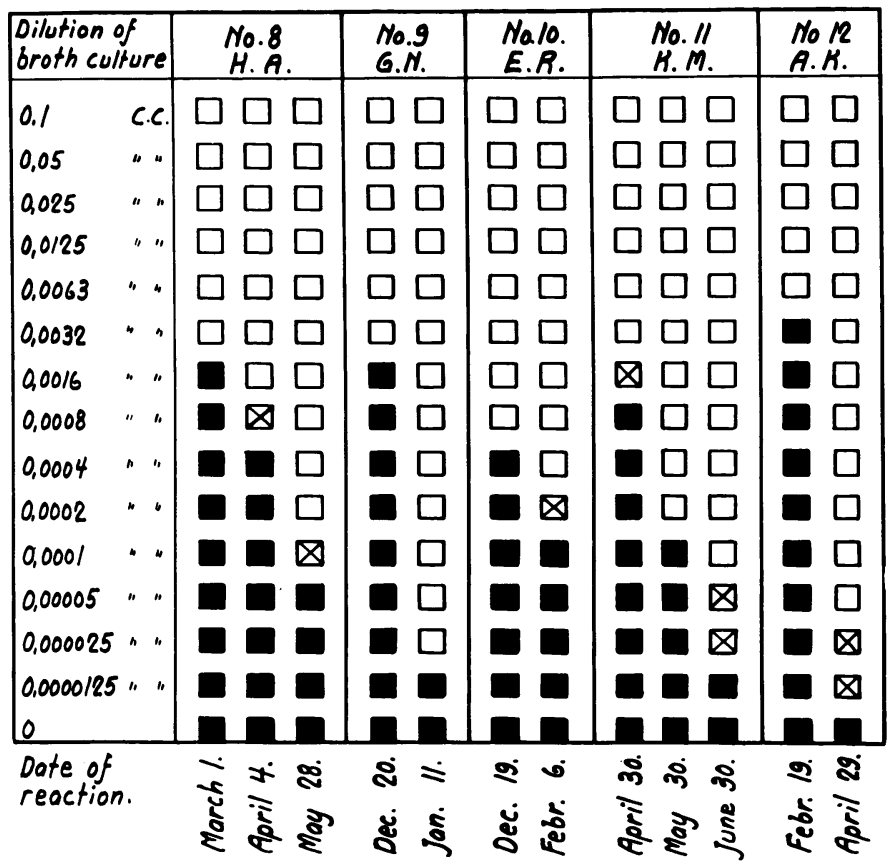

Fig. 5. Resistance to Fibrinolytic Activity in Chronic ARThritis.

pital, and the joint symptoms nearly disappeared. The antifibrinolysin reaction was positive at the time of the most active symptoms, and the susceptibility of her plasma clot became normal following improvement.

Patient Number 12, A. K. (Figure 5), 50-year old woman, for several years had had rheumatic manifestations. On arrival, she was febrile, and her sedimentation rate was $45 \mathrm{~mm}$. The electrocardiogram showed prolonged conduction time. At the time of the first test the reaction was positive, whereas her plasma clot showed a normal susceptibility two months afterward, when the symptoms of disease were less active.

Patient Number 13, B. S. (Figure 6), 26-year old woman, was admitted with a history of polyarthritis for one year. On arrival, she was febrile, with a sedimentation rate of $88 \mathrm{~mm}$. She had several infected teeth and severe carditis (heart block). The three tests which were carried out on this patient showed normal susceptibility, in spite of the fact that the joint symptoms seemed to be active, especially at the time of the first test (January 6 th).

Patient Number 14, O. K. (Figure 6), 26-year old woman, during two years had had symptoms of chronic arthritis of varying intensity. She was admitted with an involvement of several joints; afebrile; sedimentation rate $43 \mathrm{~mm}$; no carditis. Three tests were carried out, but the plasma clot was always susceptible.

In the other cases of polyarthritis, tests were performed only once during the course of the disease. The reactions given by the clots of some of these patients are shown in Figure 6. Among these cases were patients with positive and patients with negative reactions. Cases Numbers 15 and 16 had very serious and intense manifestations, and the reactions were positive, whereas the disease picture had a milder and more indistinct character in the two others in which the reactions were negative (Numbers 17 and 18).

In some of our patients there was a distinct relationship between the antifibrinolysin reaction and the clinical symptoms; and the disappearance of resistance to fibrinolytic activity coincided with the improvement. These parallel changes in the reactions and the activity of the rheumatic manifestations occurred in patients Numbers 9, 10, 11. and 12. In patient Number 8, on the other hand, the resistance disappeared without improvement in the clinical symptoms. The reaction can, however, be completely negative during a period of several months in rheumatoid arthritis. This is exemplified in the two patients, Numbers 13 and 14 , in which the activity of the clisease manifestations may, of course, have been more marked at an earlier date.

The results obtained from plasma of patients with chronic arthritis are summarized in Table I. 


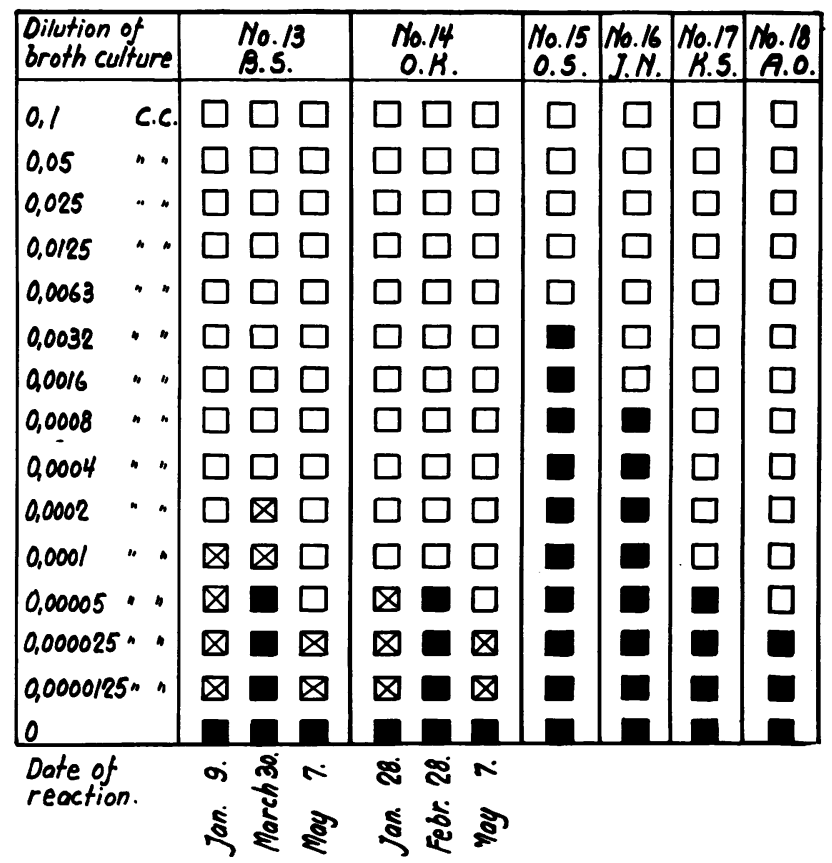

Fig. 6. Resistance to Fibrinolytic Activity in Chronic Arthritis.

TABLE I

Antifibrinolysin reactions in chronic infective arthritis

\begin{tabular}{|c|c|c|c|c|c|}
\hline \multirow{2}{*}{ Number of cases } & \multicolumn{5}{|c|}{ Type of reaction } \\
\hline & ++++ & +++ & ++ & + & 0 \\
\hline $\begin{array}{r}19 \text { adults..... } \\
5 \text { children . . }\end{array}$ & $\begin{array}{l}0 \\
0\end{array}$ & $\begin{array}{l}2 \\
0\end{array}$ & $\begin{array}{l}4 \\
0\end{array}$ & $\begin{array}{l}4 \\
0\end{array}$ & $\begin{array}{l}9 \\
5\end{array}$ \\
\hline
\end{tabular}

Five of the patients were children, 3 to 15 years old, and the disease picture in them was in most respects similar to that now known as Still's disease. They had had fever, and had had polyarthritis for. several months or years. They had active symptoms at the time the blood was collected, but apparently some of them were improving. The plasma clot of these 5 children was susceptible, so that the reactions must be characterized as negative.

Of the 19 adults, 9 had negative and 10 positive reactions. Most of the reactions were weak. Among the 9 patients who gave negative reactions, only 2 (Numbers 13 and 14) were tested several times, and some of the patients who gave a positive reaction had a normal susceptibility later on in the disease. There is, therefore, reason to believe that some of the 9 patients with the negative reactions would have shown a rise in resistance if we had been able to examine them at an earlier and probably more active stage of the disease.

\section{Bacterial endocarditis}

In this group specimens of plasma from 5 patients were tested. The disease picture corresponded in all cases to what now is known as "subacute bacterial endocarditis," or "endocarditis lenta." Among these, three had positive reactions, while the others showed normal resistance. A hemolytic fecal streptococcus was isolated from the blood of the first patient, E. H. (Figure 7). This strain did not, however, possess any fibrinolytic activity. In the 4 other patients, the disease was caused by a "Streptococcus viridans." Only two of the patients, namely F. M. and W. N., were known to have had rheumatic fever. It is at all events certain, that the two patients, E. H. and M. T., with the highest resistance, had no history of previous rheumatic attacks which could account for the positive reactions. It must, therefore, be assumed that the streptococcus found in the blood cultures had caused the rise in antifibrinolytic property of the 


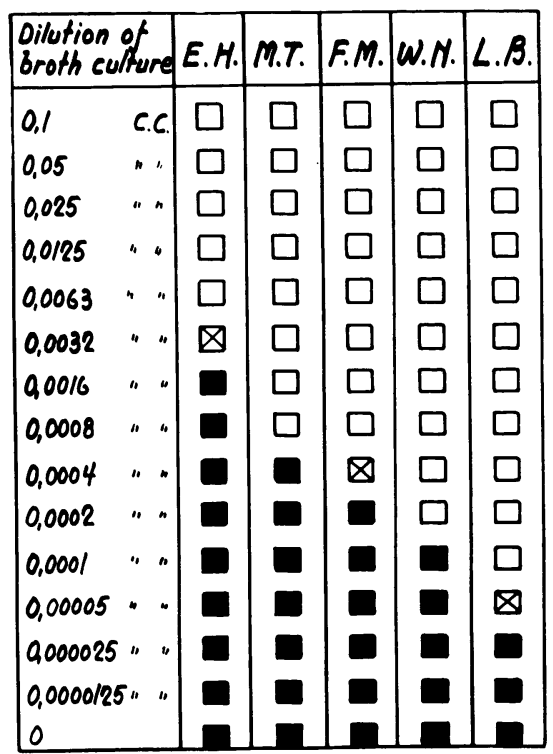

Fig. 7. Risistance to Fibrinolytic Activity in BACTERIAL ENDOCARDITIS.

plasma clot. In the patient E. H. the fecal streptococcus, which in itself lacked fibrinolytic ability, had probably given rise to a distinct antifibrinolytic reaction. The same immune response also occurred in the patient M. T., in whom green streptococcus was the etiologic agent. This is of particular interest, because Tillett and Garner (1) have found that the strains belonging to the group of nonhemolytic streptococci never possess fibrinolytic ability.

\section{DISCUSSION}

The condition in the two last mentioned cases in the group of bacterial endocarditis seems to indicate that a rise in the resistance of blood to the fibrinolytic principle is not exclusively caused by the hemolytic and fibrinolytic streptococci of the beta type. We have previously shown (6) that other groups of organisms can induce a similar elevation of the resistance; for example, the plasma clots from patients suffering from pneumonia due to pneumococci were insusceptible. The antifibrinolysin reaction is therefore not specific. As a rule, however, the highest resistance seems to develop in patients suffering from hemolytic streptococcal infections. In rheumatic fever we have found that the most marked elevation of resistance to fibrinolytic activity occurs in the se- vere cases, and that there is a close relationship between the activity of the rheumatic process and the antifibrinolysin reaction. These investigations thus support the theories of Coburn, inasmuch as Coburn and Pauli (8) have maintained that infection with toxin-producing strains of hemolytic streptococci in some way initiates the peculiar rheumatic process in susceptible subjects. The activity of the rheumatic process seems to depend upon the immune response of the rheumatic patients. The special mode, or mechanism of action of the streptococci is, however, not clear. Therefore, the question arises as to the role of the properties of the infecting organisms. Is it possible that the strains of streptococci having a low fibrinolytic ability, or none, are only to be found in cases with mild and vague manifestations? Patient Number 8 supports this probability, but further invetsigations are necessary.

It is very difficult to evaluate the results obtained in the cases of chronic infective arthritis. The degree of reaction has in 4 of the patients (Numbers 9, 10, 11, and 12) varied according to the intensity of the symptoms, and the plasma clots of 50 per cent of our patients suffering from rheumatoid arthritis showed increased resistance. The number of "positive" reactions exceeds that which Blair and Hallman (9) obtained when testing the streptolysin titer of similar patients. We consider it doubtful whether our results justify the conclusion that hemolytic streptococci are responsible for the immune response demonstrated, or that there is a significant association between these organisms and chronic infectious arthritis. This is a possibility, but our demonstration of a similar immune response in bacterial endocarditis indicates that other microbes in addition to hemolytic streptococci can cause a rise in antifibrinolytic properties of plasma clots.

At this point in our investigations we can only state that some of the patients suffering from rheumatoid arthritis give positive antifibrinolysin reactions in the active state of the disease, while others do not. To what extent the number of patients with a negative reaction can be reduced by examinations carried out at an earlier. stage of the disease remains to be seen. It would probably prove helpful in evaluating the etiologic rôle of different organisms to examine at the same 
time for the presence of agglutinins and precipitins for various streptococci and to perform antistreptolysin and antifibrinolysin tests. Great care should be taken to carry out the tests at an early stage of the disease, and to follow the reactions for a considerable period of time.

\section{CONCLUSIONS}

1. The majority of patients with rheumatic fever revealed a rise in resistance of their plasma to the fibrinolytic principle of the hemolytic streptococcus.

2. The strength of the reaction seemed to vary according to the severity of the symptoms of the disease.

3 . The susceptibility of the plasma clot returned to normal when the patients improved.

4. The plasma clots of 50 per cent of the patients suffering from chronic infective arthritis showed slight elevation of resistance to fibrinolysis. Clots from five children with Still's disease gave negative reactions.

5. In patients suffering from chronic infective arthritis the antifibrinolysin reaction may change with the activity of the disease.

6. The plasma clot of patients with bacterial endocarditis caused by fecal streptococci and green streptococci may be resistant to the fibrinolytic principle of hemolytic streptococci of the beta type.

7. The antifibrinolysin reaction is, therefore, not specific, and does not decisively prove that an infection with hemolytic streptococci of the beta type has been experienced.

The author is indebted to the doctors at the Department of Pediatrics, Rikshospitalet, Medical Department A, Rikshospitalet, Medical Department VIII and IX,
Ullevaal Hospital, and Medical Department of Akers Hospital for the supply of samples of blood from several patients.

\section{BIBLIOGRAPHY}

1. Tillett, W. S., and Garner, R. L., The fibrinolytic activity of hemolytic streptococci. J. Exper. Med., 1933, 58, 485.

2. Tillett, W. S., Edwards, L. B., and Garner, R. L., Fibrinolytic activity of hemolytic streptococci. The development of resistance to fibrinolysis following acute hemolytic streptococcus infections. J. Clin. Invest., 1934, 13, 47.

3. Tillett, W. S., The occurrence of antifibrinolytic properties in the blood of patients with acute hemolytic streptococcus infections. J. Clin. Invest., 1935, 14, 276.

4. Hadfield, G., Magee, V., and Perry, C. B., The lysis of fibrin by streptococci. Its application to the problems of rheumatic infection in children. Lancet, 1934, 226, 834.

5. Myers, W. K., Keefer, C. S., and Holmes, W. S., Jr., The resistance to fibrinolytic activity of the hemolytic streptococcus with special reference to patients with rheumatic fever and rheumatoid (atrophic) arthritis. J. Clin. Invest., 1935, 14, 119.

6. Waaler, E., Studies on the fibrinolytic activity of hemolytic streptococci and the antifibrinolytic ability of the blood from different patients. Norsk Mag. laegevidensk, 1936, 97, 449.

7. Todd, E. W., Antigenic streptococcal hemolysin. J. Exper. Med., 1932, 55, 267.

Antihemolysin titres in hemolytic streptococcal infections and their significance in rheumatic fever. Brit. J. Exper. Path., 1932, 13, 248.

8. Coburn, A. F., and Pauli, R. H., Studies on the immune response of the rheumatic subject and its relationship to activity of the rheumatic process. VI. The significance of the rise of antistreptolysin level in the development of rheumatic activity. J. Clin. Invest., 1935, 14, 769.

9. Blair, J. E., and Hallman, F. A., Streptococcal agglutinins and antistreptolysins in rheumatoid (atrophic) arthritis. J. Clin. Invest., 1935, 14, 505. 